\title{
Prediction of Icing Thickness on Transmission Lines using ANFIS model
}

\author{
AN Yuan , YAO Jiang a, ${ }^{\star}$, XI Fang ,WANG YuYao ,WEI Qian \\ Institute of Water Resources and Hydro-electric Engineering, Xi'an University \\ of technology, Xi'an 710048, Shaanxi Province, China \\ a864890175@qq.com
}

\begin{abstract}
Keywords: meteorological factors, icing process, short-term prediction, Adaptive Neural Fuzzy Inference System (ANFIS).

Abstract: Icing of transmission line is one of the most serious threats to the safe operation of power system. Its occurrence had brought great losses to the national economy. Therefore, in order to improve the reactive power and anti-risk capability of the icing power grid, it is of great significance to study the icing thickness prediction of transmission lines. In this paper, a short-term prediction method of icing process is proposed. The historical meteorological factors are used as inputs, and combined with the ice thickness increment of sampling points to train and predict the prediction model by using ANFIS. The results show that the predicted result of the short-term prediction method is effectively. And this method has higher prediction accuracy than the widely used BP neural network icing prediction.
\end{abstract}

\section{Introduction}

Ice disaster is one of the most serious threats to the power system. The ice covered on transmission line may cause the happening of some serious accident, like break line, downed towers, etc. It seriously threatens the safe operation of the power grid. There are some recorded grievous ice disaster had happened in China, America, Canada, Russia and Iceland. The ice disaster had been a common problem faced by the grid of many countries [1-3]. Especially in the early 2008, a large area of southern China suffered a rare ice disasters caused by the continued the freezing rain and snow weather. It had brought extensive damage to the power system of China and great economic losses. In order to improve the response ability and anti-risk ability of power grid to deal with icing disaster of power grid, the study of on-line monitoring, early warning and diagnosis methods for ice disaster is necessary. These can effectively help the power grid make react before the accident happened.

The icing process of transmission line is a very complicated physics process which influenced by many meteorological factors. It is difficult to explain the process through a definite analytic expression. The method that built regression model by using BP neural network algorithm is widely applied for the ice thickness prediction problem [4, 5], and achieved some results. A new short term forecast method of ice thickness was proposed. This model based on the analysis of the icing process, regarding the ambient temperature, relative humidity and wind speed as the input arguments, and achieved the prediction through ANFIS. ANFIS combine the both advantages of neural networks and fuzzy inference systems. It is very suitable for the prediction of icing thickness of transmission lines.

\section{Basis of model building}

Icing on transmission lines is a complex physical process that cold water droplets trapped by wires and frozen in cryogenic environment, the process is very complicated and influenced by many meteorological factors. By observing the historical data of icing, we found that the icing process of the transmission line is a process of constantly accumulating. The historical data of icing are $\left\{\mathrm{x}_{\mathrm{k}}, \mathrm{d}_{\mathrm{k}}\right\}$, 
$\mathrm{k}$ is the number of the sample, $\mathrm{x}_{\mathrm{k}} \in \mathrm{R}^{\mathrm{n}}$ is the corresponding meteorological data, $\mathrm{d}_{\mathrm{k}} \in \mathrm{R}$ is the corresponding icing thickness. The icing process during sample time $\Delta \mathrm{t}$ can be expressed as:

$$
\mathrm{d}(\mathrm{k}+1)=\mathrm{d}(\mathrm{k})+\Delta \mathrm{d}(\mathrm{k}) \text {. }
$$

$\mathrm{d}(\mathrm{k}+1)$ and $\mathrm{d}(\mathrm{k})$ are adjacent elements of $\mathrm{d}_{\mathrm{k}}$, and the $\Delta \mathrm{d}(\mathrm{k})$ is the increment of the ice thickness in the $\Delta \mathrm{t}$. Value of $\Delta \mathrm{d}(\mathrm{k})$ is influenced by many meteorological factors[6]. It can be seen that the ice thickness $\mathrm{d}(\mathrm{k}+1)$ is composed of ice thickness $\mathrm{d}(\mathrm{k})$ and increment of ice thickness $\Delta \mathrm{d}(\mathrm{k})$ during $\Delta \mathrm{t}$. And the entire ice process consists of several such little processes. Therefore, we have chosen to forecast ice thickness by forecasting each little icing process, and in each prediction, the previous forecast is taken as the actual value.

\section{Adaptive neural fuzzy inference system (ANFIS)}

ANFIS architecture is proposed by Jang and is developed based on the theory of fuzzy set and fuzzy logic [7]. It is a combination of two intelligence systems, namely neural network (NN) system and fuzzy inference system (FIS) in such a way that the NN learning algorithm is used to determine the parameters of the FIS. ANFIS is a type of adaptive multi-layered feed forward networks, applied to nonlinear prediction where past data samples are utilized to predict the data samples ahead. ANFIS adopts the self-learning ability of neural networks with the linguistic expression function of fuzzy inference system. A typical ANFIS architecture is given in the Figure 1 and a detailed coverage can be found in [7]. This figure shows that the structure of ANFIS with 5 layers include 1 input layer, 3 hidden layers and 1 output layer. The hidden layers represent the MFs and the fuzzy rules.

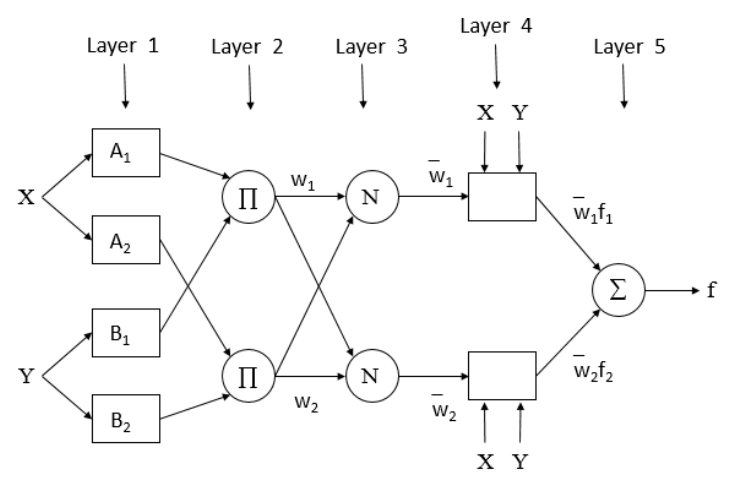

Fig. 1. AFIS architecture

The ANFIS network considered is a Takagi-Sugeno fuzzy inference system. As an example, the fuzzy if-then-rules for the first-order Sugeno-fuzzy model can be expressed as:

Rule 1: If $\mathrm{x}$ is $\mathrm{A} 1$ and $\mathrm{y}$ is $\mathrm{B} 1$, then: $\mathrm{f} 1=\mathrm{p} 1 \mathrm{x}+\mathrm{q} 1 \mathrm{y}+\mathrm{r} 1$

Rule 2: If $x$ is $A 2$ and $y$ is $B 2$, then: $f 2=p 2 x+q 2 y+r 2$

$\mathrm{x}$ and $\mathrm{y}$ are the inputs, A1, A2, B1 and B2 are the fuzzy sets, $\mathrm{f} 1$ and $\mathrm{f} 2$ are the outputs, and p1, p2, $\mathrm{q} 1, \mathrm{q} 2, \mathrm{r} 1$ and $\mathrm{r} 2$ are the parameters that are determined during the training process. The circular nodes in figure 1 represent fixed nodes, whereas the square nodes are nodes that have parameters to be learnt. Each layer in this figure is associated with a particular step in the FIS [8].

Layer 1: Each node in first layer is an adaptive node with the following node function:

$$
O_{i}^{1}=\mu_{\mathrm{A}_{i}}(x) .
$$

$x$ is the input to node $i$, and $\mathrm{A} i$ is the linguistic label associated with this node function. $O_{i}{ }^{1}$ is the membership value of a fuzzy set $\mathrm{A}_{i}$ and it indicates the degree to which the specified input $x$ satisfies the quantifier $\mathrm{A}_{i}$. For example the membership functions for $\mathrm{A}_{i}$ can be described by a generalized bell-shape MF as follows: 


$$
\mu_{\mathrm{A}_{i}}(x)=\frac{1}{1+\left[\left(\frac{x-c_{i}}{a_{i}}\right)^{2}\right]^{b_{i}}}
$$

$\left(a_{i}, b_{i}, c_{i}\right)$ is the parameter set. Parameters in this layer are said to be premise parameters.

Layer2: Each node in the second layer generates the rule firing strength via multiplication. It is marked as $\prod$ and the output is defined as:

$$
O_{i}^{2}=w_{i}=\mu_{\mathrm{A}_{i}}(x) \times \mu_{\mathrm{B}_{i}}(y) .
$$

$w_{i}(i=1,2)$ is the $i$ th rule's firing strength.

Layer 3: The number of nodes in the third layer is the same as that of the second layer. Every node $\mathrm{N}$ of this layer calculates the ratio of the $i$ th rule's firing strength to the total sum of all rules' firing strengths:

$$
O_{i}^{3}=\bar{w}_{i}=\frac{w_{i}}{w_{1}+w_{2}}, i=1,2
$$

And normalized firing strengths will be the output of this layer.

Layer 4: This layer produces fuzzy inference rules. In the fourth layer, every node refers to an adaptive node with a node function, and its output value is:

$$
O_{i}^{4}=\bar{w}_{i} f_{i}=\bar{w}_{i}\left(p_{i} x+q_{i} \mathrm{y}+r_{i}\right), i=1,2
$$

$\bar{w}_{i}$ is the output of layer 3 , and $\left(p_{i}, q_{i}\right.$ and $\left.r_{i}\right)$ is the parameter set. Parameters in this layer are said to be consequent parameters.

Layer 5: The function of the fifth layer is to perform the operations of clearness. The purpose of this layer is to convert the fuzzy numbers into clear quantities for the final output, and to compute the output of any fuzzy inference rule with weighted average. The Connection in the fifth layer is expressed in $\sum$, and the total output of the layer is:

$$
O_{i}^{5}=\text { overalloutput }=\sum_{i=1}^{2} \bar{w}_{i} f_{i}=\sum_{i=1}^{2} \bar{w}_{i}\left(p_{i} x+q_{i} \mathrm{y}+r_{i}\right) .
$$

The system structure of ANFIS belongs to a forward network with multiple network layers, the connection weight coefficients between any two layers can be trained by data objects to adjust themselves continuously. The connection weight coefficients of ANFIS networks are studied using a hybrid learning algorithm: in the forward transfer process, after a set of input data of the objects, the data is calculated to get the output of each layer of network each node by node function, then the linear least square estimation is used to identify the consequent parameters of the fuzzy rules, and the magnitude of each input output error is obtained; In the process of network reverse transmission, the method of gradient descent is used to transfer the error from the output node to the input node. At the same time, the parameters of the fuzzy rule must be adjusted constantly. Repeat operations according to the above process until the error reaches the required or set number of iterations to end the operation.

\section{Data analysis and pretreatment}

To simplify the model, we select historical data of ambient temperature, relative humidity, and wind speed as input arguments. Due to the stability and reliability of the on-line monitoring device, the original icing on-line monitoring data uploaded to the main station system inevitably have data anomalies, data omissions and other issues. To preprocess data appropriately, eliminate the abnormal data and complement the lost data, it can improve the accuracy of data analysis effectively.

1)Eliminate the abnormal data

It is generally believed that the meteorological conditions for ice icing on transmission lines are that the ambient temperature is less than or equal to $0^{\circ} \mathrm{C}$, and that the relative humidity is greater 
than or equal to $85 \%$ [9]. Affected by the breeze vibration or unstable flow, sometimes it doesn't meet the icing conditions, small icing thickness will still be monitored (often less than $2 \mathrm{~mm}$ ). It's necessary to eliminate the abnormal data for the analysis of icing effect factors.

2) Complement the lost data

The ice on-line monitoring device is set up every 15 minutes to collect a set of data and upload to the main station system. Due to the stability limit of the monitoring device on the tower or data transmission network, there will be data leakage or leakage transmission. It will cause partial realtime icing data received by the master station system loss. In order to ensure integrity and integrity of monitoring data, we used linear interpolation method to complete the data,

\section{Case study and numerical results}

We have collected the historical data of 83 office towers of Taoyi lines of Yunnan Power company from January 29, 2015 to February 1, 2015.The line monitoring system is configured to collect data of ice thickness and meteorological factors every 15 minutes. Considering the ice thickness vary little in 15 minutes, we filtered the data. Finally, an ice thickness sequence is obtained, it's time interval is $3 \mathrm{~h}$.

Table 1. Historical data of 83 office towers of Taoyi lines.

\begin{tabular}{ccccc}
\hline num & Temperature $\left[{ }^{\circ} \mathrm{C}\right]$ & Humidity $[\%]$ & Wind speed $[\mathrm{m} / \mathrm{s}]$ & Ice thickness $[\mathrm{mm}]$ \\
\hline 1 & -1.1 & 99 & 1.6 & 0.24 \\
2 & -2.5 & 99 & 1.6 & 1.20 \\
3 & -3.7 & 99 & 0.6 & 2.64 \\
4 & -4.3 & 98 & 1.6 & 3.04 \\
5 & -4.5 & 98 & 1.1 & 3.92 \\
6 & -5.1 & 98 & 0.6 & 5.12 \\
7 & -3.6 & 99 & 1.2 & 5.36 \\
8 & -1.5 & 99 & 1.6 & 5.68 \\
9 & -2.8 & 99 & 1.6 & 5.52 \\
10 & -3.6 & 99 & 1.2 & 7.04 \\
11 & -4.6 & 98 & 2.8 & 9.04 \\
12 & -5.0 & 98 & 2.6 & 10.08 \\
13 & -5.3 & 97 & 2.0 & 10.96 \\
14 & -5.3 & 97 & 1.2 & 11.76 \\
15 & -4.6 & 98 & 2.3 & 12.48 \\
16 & -1.9 & 99 & 1.5 & 13.12 \\
17 & -1.4 & 99 & 1.8 & 13.52 \\
18 & -3.3 & 99 & 1.6 & 14.24 \\
19 & -4.2 & 98 & 2.0 & 15.52 \\
20 & -4.6 & 98 & 1.2 & 16.48 \\
21 & -4.8 & 98 & 1.3 & 16.88 \\
22 & -5.0 & 98 & 2.2 & 17.28 \\
23 & -3.5 & 96 & 1.5 & 17.52 \\
\hline
\end{tabular}

The first 17 data pairs are used for training, while the others are used for checking. he number of the type chosen was to 5 and MFs for each input was set generalized triangle-shape MFs. We set parameters of training as training times is 200 , step length is $1 \mathrm{e}-3$, step growth rate is 1.25 , step down rate is 0.85 , error expectation is $1 \mathrm{e}-4$. In order to compare the differences of the traditional prediction model and this model, we respectively used BP neural network model and ANFIS icing process common forecasting model to predict the icing thickness. The parameters selection of BP neural network had shown in [8]. The forecast results of two model are shown in Table 2. 
Table 2. Prediction results of two model.

\begin{tabular}{cccc}
\hline num & $\mathrm{dk}[\mathrm{mm}]$ & $\mathrm{Bp}$ & ANFIS \\
\hline 18 & 14.24 & 13.64 & 13.48 \\
19 & 15.52 & 14.02 & 16.26 \\
20 & 16.48 & 14.42 & 15.59 \\
21 & 16.88 & 14.68 & 16.25 \\
22 & 17.28 & 14.88 & 16.87 \\
23 & 17.52 & 15.06 & 17.63 \\
\hline & RMSE & 0.117 & 0.041 \\
& MRE & $11.2 \%$ & $3.7 \%$ \\
\hline
\end{tabular}

The average relative error of ANFIS prediction model is $3.7 \%$, root mean square error is $0.041 \mathrm{~mm}$.Predicted results showed that the prediction of icing process with ANFIS has good prediction effect. And to compared with the prediction of BP neural network, this mothed's prediction capability is better.

\section{Conclusion}

(1)This paper presents a short-term prediction method of icing process based on meteorological data. We regarded the historical data of ice thickness and meteorological factors as training data, and the ice thickness prediction model is established by using ANFIS.

(2)The prediction results show that, this method has better prediction results than BP neural network. And the forecasting errors are acceptable, showing that it's very effective to predict the ice thickness by using this model. Because there are few complete icing data available for modeling and simulation, the prediction method proposed in this paper can be further improved after obtaining more complete icing data.

\section{References}

[1] M. Farzaneh. Atmospheric Icing of Power Networks[J]. 2008.

[2] X. Huang, Zhang. G. Relation of transmission line icing and local meteorology[J]. High Voltage Apparatus, 2008, 44(4): 289-294(in Chinese).

[3] X. Huang, J. Liu, C. Wei, et al. Present research situation of icing and snowing of overhead transmission lines in China and foreign countries[J]. Power System Technology, 2008, 32(4): 23-28(in Chinese).

[4] X. Huang, J. Li. Icing thickness prediction model using BP Neural Network[C]. International Conference on Condition Monitoring and Diagnosis. IEEE, 2013:758-760.

[5] X. Du, Z. Zheng, S. Tan, et al. The Study on the Prediction Method of Ice Thickness of Transmission Line Based on the Combination of GA and BP Neural Network[C]. International Conference on E-Product E-Service and E-Entertainment. IEEE, 2010:1-4.

[6] L. Makkonen. Models for the Growth of Rime, Glaze, Icicles and Wet Snow on Structures[J]. Philosophical Transactions Mathematical Physical \& Engineering Sciences, 2000, 358(1776):2913-2939.

[7] J. S. R. Jang, "ANFIS: Adaptive-Network-based Fuzzy Inference Systems," Transactions on Systems, Man, and Cybernetics. IEEE, vol. 23, no. 3, pp. 665-685, 1993.

[8] L. ZHENU, S. LIU, J. MAO, et al. A new PV generation power forecasting method integrating ANFIS and PCA[J]. Acta Energiae Solaris Sinica, 2012, 33(6):993-1001(in Chinese).

[9] X. Huang, J. LI, L. OUYANG, et al. Icing thickness prediction model using fuzzy logic theory[J]. High Voltage Engineering, 2011, 37(5): 1245-1251. 